\title{
Candidate proteomic biomarkers for three genogroups of the swine pathogen Streptococcus suis serotype 2
}

Christo Atanassov ${ }^{1,2}$, Laetitia Bonifait ${ }^{3}$, Marylise Perivier ${ }^{2}$, Marcelo Gottschalk $k^{4,5}$ and Daniel Grenier $r^{3,5^{*}}$

\begin{abstract}
Background: Streptococcus suis, more specifically serotype 2, is a major swine pathogen and an emerging zoonotic agent that causes severe infections such as meningitis, endocarditis, and septicemia. In this study, surface-enhanced laser desorption/ionization time-of-flight mass spectrometry (SELDI) was used to investigate the protein expression profiles of 45 strains of $S$. suis serotype 2 that had previously been clustered by multilocus sequence typing (MLST) into three sequence types (ST1, ST25, and ST28) ( $n=15$ for each ST).
\end{abstract}

Results: The SELDI data were analyzed using the univariate Mann-Whitney and Kruskal-Wallis tests and multivariate statistical methods (heatmap/hierarchical clustering). The heatmap identified 136 cell proteins, and hierarchical clustering provided a 100\% correct classification of all fifteen ST1 and ST25 strains and thirteen of the fifteen ST28 strains $(87 \%$ correct). The univariate statistical analyses of the SELDI protein expression profiles identified nine significant proteins that discriminated the strains of the three STs of S. suis. Of these proteins, two were overexpressed in ST1 (5958 Da and $10249 \mathrm{Da})$, four in ST25 (5989 Da, $6646 \mathrm{Da}, 7421 \mathrm{Da}$, and $9825 \mathrm{Da})$, and three in ST28 (4516 Da, $7833 \mathrm{Da}$, and $9342 \mathrm{Da}$ ). Two of the proteins associated with the ST28 strains (p4516 and p9342) were purified and were identified as a putative ABC transporter and a nucleoid-DNA-binding protein, respectively.

Conclusions: SELDI analysis of 45 strains of S. suis allowed to identify nine statistically significant proteins that can be specifically correlated with either ST1, ST25 or ST28. The possible involvement of the overexpressed proteins in the pathology of $S$. suis infections will require further investigation.

Keywords: Proteomic, SELDI, Streptococcus suis, Swine infection

\section{Background}

Streptococcus suis, more particularly serotype 2 , is a major swine pathogen and an emerging zoonotic agent. In Western countries it mainly affects individuals with occupational exposure to pigs who are in direct contact with farm animals or who handle pork meat and carcasses [1,2]. Zoonotic infections caused by S. suis are encountered more frequently in East Asia (China, Vietnam, Cambodia, Thailand, Hong Kong) where the general population is also at risk due to backyard food production

\footnotetext{
* Correspondence: daniel.grenier@greb.ulaval.ca

${ }^{3}$ Groupe de Recherche en Écologie Buccale, Faculté de Médecine Dentaire, Université Laval, 2420 de la Terrasse, Quebec City, QC G1V 0A6, Canada

${ }^{5}$ Centre de Recherche en Infectiologie Porcine et Avicole (CRIPA), Fonds de Recherche du Québec - Nature et Technologies (FRQNT), Saint-Hyacinthe, QC, Canada

Full list of author information is available at the end of the article
}

systems, wet markets, and/or consumption of raw pork meat/blood [1-3]. Porcine and human infections can be severe, with meningitis, endocarditis, and septicemia as possible clinical outcomes [3,4]. In humans, mortalities ranging from $5 \%$ to $20 \%$ have been reported $[1,5]$.

S. suis is a Gram-positive bacterium with cell wall antigenic determinants somehow related to Lancefield group D streptococci [4]. Currently, 33 serotypes have been described based on the composition of their capsular polysaccharides [2]. While the serotype distribution varies depending on the geographical origin of the strains, $S$. suis serotype 2 is considered the most pathogenic and the most prevalent capsular type among diseased pigs and humans $[2,6,7]$. Multilocus sequence typing (MLST) has shown that $S$. suis serotype 2 strains can be divided into different sequence types (STs) [8]. Closely related STs are grouped in ST complexes, and several dominate 
the S. suis population, including ST1, ST16, ST25, ST28, and ST147. Most invasive strains causing human outbreaks in Europe and Asia are ST1 strains [9-11] or, at least, belong to the ST1 and ST16 complexes $[8,12-14]$. This is in agreement with the recent study by Fittipaldi et al. [15], who showed, using a mouse infection model, that European ST1 strains are highly virulent while North American ST25 strains display moderate virulence and North American ST28 strains are weakly virulent.

The reasons for these differences in virulence are not well understood [15] while the discovery of novel candidate virulence factors that are either produced exclusively or overexpressed by ST1 strains is a major theoretical and practical issue. The search for putative $S$. suis virulence factors has been conducted using genomic [16-22] and proteomic tools [23-31]. Proteomic studies of $S$. suis are based on the initial separation of bacterial proteins by two-dimensional polyacrylamide gel electrophoresis (2D PAGE) followed by the enzymatic digestion of selected protein bands and the identification of the resulting peptides by mass spectrometry combined with bio-informatics analyses of databases of sequenced genomes from this taxon. While proteomic methods have made a major contribution, they suffer from limitations inherent to 2D electrophoresis, which often fails to detect some proteins, especially those with small molecular weights or extreme isoelectric points. These limitations can be overcome using surface-enhanced laser desorption/ionization time-of-flight mass spectrometry (SELDITOF-MS; SELDI ProteinChip), a medium-throughput technology that allows protein expression levels in hundreds of samples in a single experiment to be compared $[32,33]$. We used this approach to analyze $45 \mathrm{~S}$. suis serotype 2 strains and found nine statistically significant proteins correlated to the three STs that had previously been identified by MLST (ST1, ST25, and ST28). Moreover, two of these biomarkers were purified and identified.

\section{Methods}

\section{Bacterial strains}

Forty-five S. suis serotype 2 strains isolated from diseased pigs or humans from different countries were included in the SELDI analysis. They were either ST1, ST25, or ST28 strains (see Table 1) based on previous MLST analyses ([15], unpublished data).

\section{Preparation of bacterial cells}

All S. suis strains were grown in parallel at $37^{\circ} \mathrm{C}$ in $10 \mathrm{ml}$ of Todd-Hewitt broth (BBL Microbiology Systems, Cockeysville, MD, USA) until they reached an optical density $(660 \mathrm{~nm})$ in the range of 0.4 to 0.5 corresponding to the mid-log phase. The bacterial cells were harvested by centrifugation at 3,000 $\times g$ for $10 \mathrm{~min}$ at $4^{\circ} \mathrm{C}$. The cell pellets were washed three times in
$50 \mathrm{mM}$ phosphate-buffered saline (pH 7.4) (PBS) supplemented ex tempore with $2 \mathrm{mM} 4$-(2-aminoethyl)benzenesulfonyl fluoride hydrochloride (AEBSF; Sigma-Aldrich Canada, Oakville, ON, Canada). After centrifugation at $3,000 \times g$ for $10 \mathrm{~min}$ at $4^{\circ} \mathrm{C}$, the cell pellets were immediately stored at $-80^{\circ} \mathrm{C}$ until used.

\section{Protein extraction}

The frozen cell pellets were thawed and were individually suspended in $1 \mathrm{ml}$ of lysis buffer $\left(16 \mathrm{mM} \mathrm{Na}_{2} \mathrm{HPO}_{4}\right.$, $4 \mathrm{mM} \mathrm{NaH}_{2} \mathrm{PO}_{4}, 150 \mathrm{mM} \mathrm{NaCl}, 1 \%$ Triton X-100) supplemented with cOmplete protease inhibitor cocktail (Roche Diagnostics, Laval, QC, Canada). The suspensions were transferred into FastPROTEIN BLUE tubes and were homogenized using a FastPrep apparatus (MP Biomedicals, Solon, OH, USA). The suspensions were homogenized using six cycles (40 s each) at power setting 6 , with a 5-min cooling period on ice between each cycle. The homogenates were then centrifuged at $15,000 \times g$ for $15 \mathrm{~min}$, and the supernatants were divided into aliquots and stored at $-80^{\circ} \mathrm{C}$ until used.

\section{ProteinChip array processing}

Two types of ProteinChip ion-exchange arrays (Q10 and CM10; Bio-Rad Laboratories, Mississauga, ON, Canada) were assembled in a 96-well bioprocessor (Bio-Rad Laboratories) and were preactivated for $30 \mathrm{~min}$ with their respective buffers (100 mM Tris- $\mathrm{HCl}, \mathrm{pH}$ 9.0, and $100 \mathrm{mM}$ sodium acetate, $\mathrm{pH}$ 4.0, respectively). Binding buffers $(180 \mu \mathrm{l})$ for the respective arrays were then mixed with $20 \mu \mathrm{l}$ of protein extract adjusted to a final protein concentration of $0.5 \mathrm{mg} / \mathrm{ml}$, and the mixtures were incubated for $60 \mathrm{~min}$. All the protein samples were tested in duplicate. After two washes with binding buffer and one quick rinse with HPLC-grade water the spots were loaded twice with $1 \mu \mathrm{l}$ of a saturated solution of sinapinic acid dissolved in 50\% acetonitrile $(\mathrm{ACN}) / 0.5 \%$ trifluoroacetic acid (TFA) (v/v). All the steps were carried out at room temperature $\left(18-20^{\circ} \mathrm{C}\right)$ using a Micromix-5 platform shaker and a Biomek 3000 robot-pipetting workstation (Beckman-Coulter France, Villepinte, France). The arrays were processed using a PCS 4000 ProteinChip Reader (Bio-Rad Laboratories) programmed in positive ion mode with an ion acceleration potential of $20 \mathrm{kV}$.

\section{Spectra processing and statistical analysis}

Once the spectra were calibrated and normalized using the total ion current method, clusters of peaks with the same mass were defined using the following settings: $\mathrm{S} / \mathrm{N}$ (first pass) $\geq 5$, minimum peak threshold: $20 \%$, mass error: $0.3 \%, \mathrm{~S} / \mathrm{N}$ (second pass) $\geq 2$. Three types of computergenerated statistics performed using ProteinChip Data Manager 3.0.7 software (Bio-Rad Laboratories) were used to analyze the data (the non-parametric Mann-Whitney $\mathrm{U}$ 
Table 1 Characteristics of S. suis strains serotype 2 used in the SELDI analysis

\begin{tabular}{|c|c|c|c|c|}
\hline Strains & ST & Country & Province/State & Infection \\
\hline $\mathrm{P} 1 / 7$ & $1^{*}$ & Europe & $N A^{* * * *}$ & Meningitis \\
\hline MNCM01 & 1 & Thailand & Chiang Mai & Endocarditis \\
\hline MNCM06 & 1 & Thailand & Chiang Mai & Meningitis \\
\hline MNCM16 & 1 & Thailand & Chiang Mai & Meningitis \\
\hline MGGUS2 & 1 & United States & Wisconsin & Meningitis \\
\hline MGGUS3 & 1 & United States & lowa & Meningitis \\
\hline NIAH11433 & 1 & Japan & NA & Meningitis \\
\hline DAT261 & 1 & Japan & Gunma & Pneumonia \\
\hline DAT264 & 1 & Japan & Gunma & Meningitis \\
\hline DAT229 & 1 & Japan & Aichi & Endocarditis \\
\hline P 517/03 P4 & 1 & Argentina & Buenos Aires & Meningitis \\
\hline P 477/03 P1 & 1 & Argentina & Cordoba & Meningitis \\
\hline P 613/05 P3 & 1 & Argentina & Buenos Aires & Meningitis \\
\hline 31533 & 1 & France & Brittany & Meningitis \\
\hline SS166 & 1 & France & Brittany & Meningitis \\
\hline 1043248 & $25^{* *}$ & Canada & Quebec & Meningitis \\
\hline 1043629 & 25 & Canada & Quebec & Pneumonia \\
\hline 1044423 & 25 & Canada & Ontario & NA \\
\hline 1053253 & 25 & Canada & Manitoba & Pneumonia \\
\hline 1085543 & 25 & Canada & Quebec & Meningitis \\
\hline MNCMO4 & 25 & Thailand & Chiang Mai & Meningitis \\
\hline MNCM10 & 25 & Thailand & Chiang Mai & Septicemia \\
\hline MNCM24 & 25 & Thailand & Chiang Mai & Endocarditis \\
\hline MNCM26 & 25 & Thailand & Chiang Mai & Meningitis, endocarditis \\
\hline MNCM51 & 25 & Thailand & Chiang Mai & Septicemia \\
\hline MGGUS4 & 25 & United States & lowa & Septicemia \\
\hline 1102864 & 25 & Canada & Quebec & Septicemia \\
\hline 1102337 & 25 & Canada & Quebec & Meningitis \\
\hline LPH4 & 25 & Thailand & Lamphun & Septicemia \\
\hline LPH12 & 25 & Thailand & Lamphun & Septicemia \\
\hline 1054471 & $28^{* * *}$ & Canada & Manitoba & Meningitis \\
\hline 1088563 & 28 & Canada & Quebec & Meningitis \\
\hline 1097205 & 28 & Canada & Ontario & Meningitis \\
\hline 1057906 & 28 & Canada & Saskatchewan & Meningitis \\
\hline MNCM43 & 28 & Thailand & Chiang Mai & Endocarditis \\
\hline MGGUS9 & 28 & United States & Oklahoma & Endocarditis \\
\hline MGGUS10 & 28 & United States & Illinois & Pneumonia \\
\hline MGGUS11 & 28 & United States & Virginia & Pneumonia \\
\hline MGGUS12 & 28 & United States & lowa & Pneumonia \\
\hline DAT242 & 28 & Japan & Ibaraki & Meningitis \\
\hline DAT245 & 28 & Japan & Ibaraki & Meningitis \\
\hline DAT246 & 28 & Japan & Ibaraki & Septicemia \\
\hline
\end{tabular}


Table 1 Characteristics of S. suis strains serotype 2 used in the SELDI analysis (Continued)

\begin{tabular}{llll}
\hline $\mathbf{1 0 8 4 7 0 8}$ & 28 & Canada & Ontario \\
PAH-17 & 28 & United States & Minnesota \\
DAT251 & 28 & Japan & Ishikawa \\
\hline
\end{tabular}

*, All ST1 strains are $m r p+$, ef+, sly+; ${ }^{* *}$, All ST25 strains are $m r p-$, ef-, sly-; ${ }^{* * *}$, All ST28 strains are $m r p+$, ef-, sly-; ${ }^{* * * *}$, Not available.

test, the Kruskal-Wallis $\mathrm{H}$ test, and the heatmap/hierarchical clustering method).

\section{Protein purification and identification}

The protein extracts were dialyzed overnight at $4^{\circ} \mathrm{C}$ against a 1,000-fold volume of $20 \mathrm{mM}$ Tris- $\mathrm{HCl}$ ( $\mathrm{pH} 9.5)$. They were then fractionated by ion-exchange chromatography (IEX) using HiTrap Q HP columns (GE Healthcare Life Sciences, Velizy-Villacoublay, France) as described previously [34]. IEX fractions containing the target proteins were further separated by reversed-phase high pressure liquid chromatography (RP-HPLC; GE Healthcare Life Sciences) according to Lanotte et al. [34]. The RPHPLC fractions were concentrated 20 -fold in a vacuum centrifuge (miVac, Genevac, Ipswich, England) to a final volume of $\sim 50 \mu \mathrm{l}$. Thereafter, $3 \mu \mathrm{l}$ of the concentrate was spotted on gold arrays (Bio-Rad Laboratories) and was analyzed in the MALDI mode using a SELDI PCS 4000 apparatus and the following acquisition protocol: focus mass 10,000; laser energy 3,000; matrix attenuation 2,500; partition $1 / 1 ; 20$ shots. The fraction containing the target protein was dried completely in a vacuum centrifuge and was then reconstituted in tricine sodium dodecyl sulfate (SDS) sample buffer containing NuPAGE reducing agent (Invitrogen SARL, Cergy, France). The mixture was divided into two samples that were heated at $40^{\circ} \mathrm{C}$ for $30 \mathrm{~min}$ and separated in parallel on 1D home-cast Tris-Tricine gels $(18 \% \mathrm{~T} / 6 \% \mathrm{C})$ at $30 \mathrm{~V}$ constant for $1 \mathrm{~h}$ followed by $60 \mathrm{~mA}$ constant for an additional $15 \mathrm{~h}$. Three lanes (two lateral and one in the middle of the gel) were loaded with prestained molecular weight (MW) markers, which served to indicate the approximate position of the target proteins in the unstained gel. One-mm-thick gel slices covering two adjacent lanes containing the same protein were excised. Each gel slice was further divided into two equal parts corresponding to one separate lane. One of the slices was passively eluted to extract the target protein as described previously [35]. Once the mass of the passively eluted target protein was confirmed on the gold array, the protein in the corresponding second slice was trypsin digested and LC-MS/MS microsequenced as described previously [34].

\section{Database search and processing of results}

Data were searched using SEQUEST through a Bioworks 3.1.1 interface (ThermoFinnigan, San Jose, CA, USA) against a subset of the NCBI non-redundant database restricted to $S$. suis entries (http://www.ncbi.nlm.nih. gov/genome/genomes/199). Peak lists were extracted using extract-msn (BioWorks 3.3.1 Thermo Scientific) using the default settings. DTA files were generated from MS/MS spectra that reached a minimal intensity ( $\mathrm{n}=$ $100)$ and a sufficient number of ions $(n=5)$. The DTA files allowed several MS/MS spectra corresponding to the same precursor ion with a tolerance of $50 \mathrm{ppm}$ to be averaged. Spectra from peptides with molecular masses between $600 \mathrm{Da}$ and 4,500 Da were retained. The search parameters were as follows: the mass accuracy of the monoisotopic peptide precursor was set at $10 \mathrm{ppm}$ and the mass accuracy of the peptide fragments was set at $0.5 \mathrm{amu}$. Only b-ions and y-ions were considered for the mass calculations. The oxidation of methionine (+16 Da) was considered as a variable modification. Two missed trypsin cleavages were allowed. Only peptides with Xcorr values higher than 2.0 (double charge), 2.5 (triple charge), and 3.0 (more than 3 charges) were retained. In all cases, the peptide $\mathrm{p}$-value was required to be below 0.001 and the DeltaCn value to be above 0.1. All protein identifications were based on the detection of a minimum of two distinct peptides. No false positives were detected using these parameters. Shared peptides were only counted for the proteins that had the most matching peptides.

\section{Results}

The protein expression profiles of 45 S. suis serotype 2 strains obtained on two types of ProteinChip arrays (CM10 and Q10) were submitted to multivariate heatmap/hierarchical clustering and univariate analyses. Hierarchical clustering was based on two main spectral features of each detected protein: a qualitative feature $(\mathrm{m} /$ $\mathrm{z}$ or mass-to-charge ratio; here $\mathrm{z}=1$, which means that $\mathrm{m} /$ $\mathrm{z}$ directly reflects the mass [expressed in Daltons]) and a semi-quantitative feature (intensity, expressed in $\mu \mathrm{A}$ per laser pulse). Hierarchical clustering of the patterns was generated with respect to the following criteria: (i) sequence type (ST1 versus ST25 versus ST28), (ii) continent of origin (Far East Asia, including Japan and Thailand versus North America, including Canada and USA), (iii) country of origin (Canada versus Japan versus Thailand versus USA), and (iv) type of infection and pathological lesion (Group A - endocarditis/pneumonia/septicemia versus Group B - meningitis). 
While the clustering of $S$. suis protein profiles by continent, country, and pathology was unsuccessful (see Additional file 1: Figure S1, Additional file 2: Figure S2 and Additional file 3: Figure S3), the clustering of S. suis protein profiles by ST generated a very high level of correct classification (Figure 1). The detailed view of the heatmap on Figure 1 shows 136 proteins in the three groups of S. suis strains defined by ST (ST1, ST25, and ST28). Interestingly, hierarchical clustering provided a $100 \%$ correct classification of all fifteen ST1 and ST25 strains and thirteen of the fifteen ST28 strains (87\% correct classification).

Some combinations of biomarkers (arbitrarily delimited by yellow- and grey-bordered rectangles on Figure 1) made it possible to delineate the groups from each other. However, none of the combinations was completely homogenous in terms of over-expression (or upregulation) or under-expression (or down-regulation) (red and green boxes in the respective rectangles). Another practical consideration was that the number of candidate biomarkers turned out to be too large to be of use for further purification and identification in academic laboratories such as ours. In addition, while multivariate clustering has the advantage of providing a global overview of the profiles, it does not allow a precise quantification of expression. In practice, the usual colors of the rectangles on the heatmaps (red standing for over-expression or up-regulation, green for underexpression or down-regulation, and black for no difference) are nuanced as more or less dark.

The same spectra were analyzed by the Mann-Whitney (applicable to two groups of data sets only) and KruskalWallis (applicable to more than two groups; in our study three or four) tests to limit the number of the candidate biomarkers and to evaluate their expression levels more precisely while trying to select the most statistically discriminating ones for further purification and sequencing. The statistical comparisons were made point-by-point, that is, for candidate biomarkers with the same mass whose statistically significant variations in intensity were calculated either for S. suis groups defined by their ST or other criteria such as strain origin and pathology. Four selection criteria with respect to the $S$. suis strains that were discriminated by their ST (Figure 2) as well as by continent, country of origin, or pathology (Additional file 4: Table S1, Additional file 5: Table S2 and Additional file 6: Table S3) were compared using a multivariate analysis. However, unlike the multivariate analysis where discriminated protein profiles were correlated only with the ST of the $S$. suis strains, the univariate Mann-Whitney and Kruskal-Wallis analyses revealed candidate biomarkers in all cases. It should be noted that only the most statistically significant candidate biomarkers in Figure 2 and in Additional file 4: Table S1, Additional file 5: Table S2 and Additional file 6: Table S3 are presented (9 in Figure 2; 24, 30, and 12 in Additional file 4: Table S1, Additional file 5: Table S2 and Additional file 6: Table S3, respectively). Figure 2 shows the nine most discriminating proteins found in the S. suis strains divided by ST. Of these, two were overexpressed in ST1 (5,958 Da and 10,249 Da), four in ST25 (5,989 Da, 6,646 Da, 7,421 Da, and 9,825 Da), and three in ST28 (4,516 Da, 7,833 Da, and 9,342 Da). Interestingly, only two of the biomarkers in Figure 2 were found statistically significant when all strains were compared by combining ST and type of pathology in two groups: group A (heart/lung/sepsis) and group B (meningitis/brain). The first is p5958, which was overexpressed in the S. suis ST28 strains (Figure 2), as well as in strains isolated from pigs/humans with meningitis (Additional file 6: Table S3). The second is p5989, which was overexpressed in the S. suis ST1 strains (Figure 2) and in strains isolated from pigs/humans with cardiac and pulmonary pathologies or sepsis (Additional file 6: Table S3).

As a next step, we tried to purify and identify the most promising candidate biomarkers. They were purified using several purification steps, including IEX, RPHPLC, and 1D SDS-PAGE. All the fractions obtained throughout the purification procedure were systematically tested for the presence of target proteins by SELDI (IEX fractions), MALDI (RP-HPLC fractions, and passive elution (1D SDS-PAGE gel slices). The purified proteins were trypsinized, identified by LC-MS/MS, and submitted to an in silico comparison of the masses of the peptides to the masses in the proteome database of $19 \mathrm{~S}$. suis reference strains with completely sequenced genomes and 75 strains from the same taxon whose genomes are currently being sequenced (http://www.ncbi. nlm.nih.gov/genome/genomes/199). We eventually succeeded in identifying two of the proteins (p4516 and p9342) as a putative ATP-binding cassette (ABC) transporter and a nucleoid-DNA-binding protein, respectively.

\section{Discussion}

Compared to studies that are usually performed on a limited number of bacterial strains, population-based studies seem to be a more appropriate approach for identifying species-specific biomarkers. Our study on a population of $45 \mathrm{~S}$. suis serotype 2 strains is thus more representative of species whose main genotypes have already been defined by MLST. The search for candidate biomarkers has benefited from the advent of mass spectrometry, which has also proven to be a promising and reliable approach for classifying bacteria. Several techniques are currently being used, including MALDI-TOFMS, LC-ESI-MS, and SELDI-TOF-MS [32-35]. When applied to the characterization of complex protein samples (bacterial extracts, for example), approximately 100 proteins can be detected by MALDI and SELDI while 


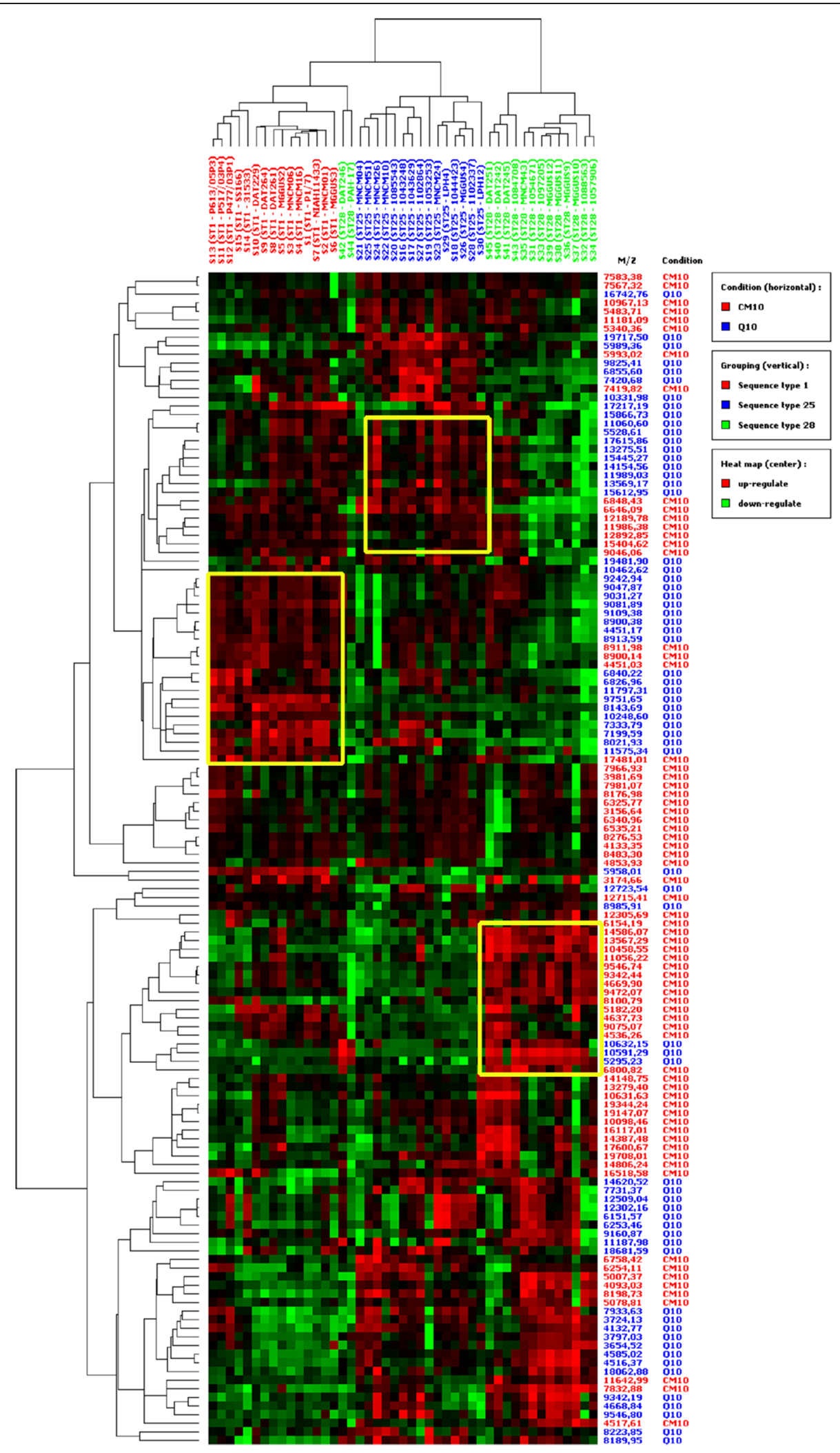

Figure 1 (See legend on next page.) 
(See figure on previous page.)

Figure 1 Heatmap/hierarchical clustering of 136 proteins of 45 S. suis strains discriminated by MLST into three sequence type (ST) groups: ST1 $(n=15)$, ST25 $(n=15)$, and ST28 $(n=15)$. The clusters were obtained by combining the average intensity values of all samples tested in duplicate on CM10 and Q10 ProteinChip arrays (acquisition protocol 1). The consecutive numbers of each strain used in the SELDI expression difference mapping (EDM) are indicated above the image (S1-S15 for the ST1 group (in red); S16-S30 for the ST25 group (in blue), and S31-S45 for the ST28 group (in green) followed by the respective sequence types and original names of the S. suis strains (in brackets). The protein masses detected on the CM10 (red) and Q10 (blue) ProteinChip arrays are indicated on the right side of the image. Specific EDM conditions: First pass: peak $S / N \geq 5$, valley depth $S / N \geq 3$, minimal peak threshold - 20\% for all spectra; second pass: peak $S / N \geq 2$, valley depth $S / N \geq 2$; third pass: adding estimated (missing) peaks to complete the clusters, clustered mass window width $-0.1 \%$, autocentroid marks on peaks, $M / Z$ range of analysis ( $z=1): 3000-20000 \mathrm{Da}$.

more than 500 proteins can typically be detected by LCESI-MS. However, LC-ESI-MS has the reputation of being labor intensive and time consuming and of requiring multiple replicates [36]. In our study, we used SELDI in which proteins and their fragments are characterized by their $m / z$, and their abundance is estimated in a semiquantitative manner by the level of protein expression. The limitations of the present study arise from the fact that it does not take into consideration extracellular proteins as well as cell proteins that are not expressed under the bacterial growth conditions used. In addition, SELDI is more adapted for proteins with a molecular mass of less than $30 \mathrm{kDa}$.

The EDM of proteins based on their relative abundance (as deduced from measured values of $\mu \mathrm{A}$ laser/ pulse) was done in extracts from bacterial cells grown in parallel under the same culturing condition, without any specific induction of protein expression. All tested $45 \mathrm{~S}$. suis strains were divided in three groups by sequence type and the level of average protein expression was evaluated in a comparative way, within the frame of these three STs. It is noteworthy that the level of expression of a particular protein, measured in one ST group of fifteen strains, could in fact represent a normal level for this ST, but be considered as high and/or low compared to the two other ST groups.

Two peptides that were overexpressed in ST28 strains (compared to those of ST1 and ST25 groups) were purified and identified, p4516 and p9342. The first one, p4516, was a fragment of an uncharacterized protein of S. suis, i.e. "hypothetical protein Ssui0_06242 [Streptococcus suis 05HAS68]". Its BLAST analysis revealed sequence homology (ca. $40 \%$ identity) with a family of ATP-dependent transmembrane proteins involved in the translocation of substrates across the bacterial membrane. Interestingly, except for S. suis 05HAS68 where this putative $A B C$ transporter was identified, sequence homologies were all related to $\mathrm{ABC}$ transporters of other streptococci taxa, such as $S$. agalactiae and $S$. oralis (Additional file 7: Table S4). The second one, p9342, was a fragment of a putative nucleoid DNA-binding protein, which may play a role in genome maintenance and gene

\begin{tabular}{|c|c|c|c|c|c|c|c|c|c|c|c|}
\hline \multirow{3}{*}{$\begin{array}{l}\text { Biomarker } \\
\text { Mass (Da) }\end{array}$} & \multirow{3}{*}{$\begin{array}{c}\text { Protein } \\
\text { array }\end{array}$} & \multirow{3}{*}{$\begin{array}{c}\text { ST1 } \\
\begin{array}{c}\text { Average } \\
\text { intensity }\end{array}\end{array}$} & \multirow{3}{*}{$\begin{array}{c}\text { ST25 } \\
\begin{array}{c}\text { Average } \\
\text { intensity* }\end{array}\end{array}$} & \multirow{3}{*}{$\begin{array}{c}\text { ST28 } \\
\begin{array}{c}\text { Average } \\
\text { intensity* }\end{array}\end{array}$} & \multirow{3}{*}{\begin{tabular}{|c|} 
Kruskal-Wallis test \\
$\begin{array}{c}\text { (ST1 vs. ST25 vs. } \\
\text { ST28) }\end{array}$ \\
P value \\
\end{tabular}} & \multicolumn{6}{|c|}{ Mann-Whitney test } \\
\hline & & & & & & \multicolumn{2}{|c|}{ ST1 vs. ST25 } & \multicolumn{2}{|c|}{ ST1 vs. ST28 } & \multicolumn{2}{|c|}{ ST25 vs. ST28 } \\
\hline & & & & & & $P$ value & $A U C^{\star \star}$ & $P$ value & $A U C^{\star \star}$ & $P$ value & $A U C^{\star \star \star}$ \\
\hline 4516.37 & Q10 & 9.53 & 17.53 & 35.74 & 0.00000145 & 0.00045678 & 0.85 & 0.00000558 & 0.98 & 0.00449374 & 0.79 \\
\hline 5958.01 & Q10 & 3.98 & 0.26 & 0.72 & 0.00000074 & 0.00003674 & 0.07 & 0.00007459 & 0.07 & 0.00028416 & 0.87 \\
\hline 5989.36 & Q10 & 1.69 & 4.67 & 2.71 & 0.00000056 & 0.00001209 & 0.95 & 0.00028416 & 0.90 & 0.00028416 & 0.15 \\
\hline 6646.09 & CM10 & 17.22 & 32.58 & 1.95 & 0.00000008 & 0.00659138 & 0.77 & 0.00000307 & 0.02 & 0.00000307 & 0.02 \\
\hline 7420.68 & Q10 & 3.95 & 6.44 & 2.51 & 0.00011761 & 0.02378685 & 0.71 & 0.02943594 & 0.26 & 0.00003674 & 0.10 \\
\hline 7832.88 & CM10 & 4.49 & 7.36 & 21.13 & 0.00007149 & 0.02648151 & 0.74 & 0.00033341 & 0.87 & 0.00053336 & 0.87 \\
\hline 9342.44 & CM10 & 46.99 & 36.52 & 64.99 & 0.00034480 & 0.01910321 & 0.26 & 0.00844210 & 0.77 & 0.00053336 & 0.85 \\
\hline 9825.41 & Q10 & 1.94 & 3.00 & 1.36 & 0.00000993 & 0.01074448 & 0.77 & 0.00045678 & 0.13 & 0.00004397 & 0.07 \\
\hline 10248.60 & Q10 & 3.12 & 1.91 & 0.98 & 0.00000134 & 0.00130649 & 0.18 & 0.00000307 & 0.02 & 0.00302013 & 0.21 \\
\hline
\end{tabular}

\footnotetext{
* Average intensity: values expressed in $\mu \mathrm{A}$ laser/pulse

** ST1 as group 0 when calculating AUC (area under the curve in receiver operator characteristic [ROC] plots)

${ }^{\star * \star} \mathrm{ST} 25$ as group 0 when calculating AUC

Red background and fonts in bold - highest average intensity indicating overexpression

D Orange background - intermediary level of average intensity

Light green background - lowest average intensity indicating underexpression

Highest $P$ value across all Mann-Whitney tests serving as a threshold for all others (i.e., $P \leq 0.0294$ )
}

Figure 2 Candidate biomarkers of $S$. suis selected by univariate analysis of two or three groups of strains divided by sequence type. 
expression regulation. BLAST analysis showed that this nucleoid DNA-binding protein is very highly conserved across different S. suis clinical isolates (100\% identity in $17 \mathrm{~S}$. suis strains), and, to a lesser extent, in other streptococci and lactobacilli (Additional file 7: Table S4). Further studies are required to determine why these proteins are over-expressed by less virulent strains of S. suis. One hypothesis is that they may be the products of antivirulence genes, which are known to interfere with bacterial virulence [37]. For instance, Zhao et al. [38] identified the ArgT protein as an anti-virulence factor since it interferes with the virulence of Shigella flexneri by transporting specific amino acids or by modulating the expression of the protease HtrA.

\section{Conclusions}

In summary, SELDI analysis of 45 strains of $S$. suis allowed to identify nine statistically significant proteins that can be specifically correlated with either ST1, ST25 or ST28. The possible involvement of the overexpressed proteins in the pathology of $S$. suis infections will require further investigation.

\section{Additional files}

Additional file 1: Figure S1. Protein profiles of S. suis strains divided by continent of origin: Far East (Japan and Thailand) versus North America (Canada and USA).

Additional file 2: Figure S2. Protein profiles of S. suis strains divided by country of origin: Canada versus Japan versus Thailand versus USA.

Additional file 3: Figure S3. Protein profiles of S. suis strains divided by type of infection/pathological lesion: group A (endocarditis/pneumonia/ septicemia) versus group B (meningitis).

Additional file 4: Table S1. Candidate biomarkers of S. suis selected by univariate analysis (Mann-Whitney test): comparison by continent of origin.

Additional file 5: Table S2. Candidate biomarkers of S. suis selected by univariate analysis (Kruskal-Wallis test) of 4 groups of strains divided by country of origin.

Additional file 6: Table S3. Candidate biomarkers of S. suis selected by univariate analysis (Mann-Whitney test) of two groups of strains divided by type of pathological lesion.

Additional file 7: Table S4. Essential sequence and BLAST data of the two proteins identified through p4516 (putative $A B C$ transporter) and p9342 (nucleoid-binding protein).

\section{Competing interests}

The authors declare that they have no competing interests.

\section{Authors' contributions}

CA, MP, and LB performed all the experimental work. CA, MG, and DG conceived the study design and prepared the final version of the manuscript. All authors read and approved the final manuscript.

\section{Acknowledgments}

This study was supported by grants from the Natural Sciences and Engineering Research Council of Canada (NSERC) to MG and DG. We also wish to thank Dr. Daisuke Takamatsu (National Institute of Animal Health, Tsukuba, Ibaraki, Japan) and Dr. Prasit Tharavichitkul (Chiang Mai University, Chiang Mai, Thailand) for providing some of the strains used in this study. Special thanks to Dr. Stephane Claverol (Pôle Protéomique, Centre de
Génomique Fonctionnelle, Université Victor Segalen - Bordeaux 2, France) for LC-MS/MS sequencing.

\section{Author details}

${ }^{1}$ Laboratoire de Bactériologie-Hygiène, Centre Hospitalier Universitaire de Poitiers, Poitiers, France. ²EA 4331 LITEC, Pôle Biologie-Santé, Université de Poitiers, Poitiers, France. ${ }^{3}$ Groupe de Recherche en Écologie Buccale, Faculté de Médecine Dentaire, Université Laval, 2420 de la Terrasse, Quebec City, QC G1V 0A6, Canada. ${ }^{4}$ Groupe de Recherche sur les Maladies Infectieuses du Porc, Faculté de Médecine Vétérinaire, Université de Montréal, Saint-Hyacinthe, QC, Canada. ${ }^{5}$ Centre de Recherche en Infectiologie Porcine et Avicole (CRIPA), Fonds de Recherche du Québec - Nature et Technologies (FRQNT), Saint-Hyacinthe, QC, Canada.

Received: 15 April 2014 Accepted: 10 March 2015

Published online: 04 April 2015

\section{References}

1. Gottschalk M, Xu J, Calzas C, Segura M. Streptococcus suis: a new emerging or an old neglected zoonotic pathogen? Future Microbiol. 2010;5:371-91.

2. Gottschalk M, Segura M, Xu J. Streptococcus suis infections in humans: the Chinese experience and the situation in North America. Anim Health Res Rev. 2007:8:29-45.

3. Huong VT, Ha N, Huy NT, Horby P, Nghia HD, Thiem VD, et al. Epidemiology, clinical manifestations, and outcomes of Streptococcus suis. Emerg Infect Dis. 2014;20:1105-14.

4. Gottschalk M. Streptococcosis. In: Karriger LRA, Schwartz KJ, Stevenson G, Zimmerman J, editors. Diseases of swine. NJ, USA: Wiley Publishers; 2012. p. 841-55.

5. Huang YT, Teng LJ, Ho SW, Hsueh PR. Streptococcus suis infection. J Microbiol Immunol Infect. 2005;38:306-13.

6. Messier S, Lacouture S, Gottschalk M. Distribution of Streptococcus suis capsular types from 2001 to 2007. Can Vet J. 2008;49:461-2.

7. Wei Z, Li R, Zhang A, He H, Hua Y, Xia J, et al. Characterization of Streptococcus suis isolates from the diseased pigs in China between 2003 and 2007. Vet Microbiol. 2009;137:196-201.

8. King SJ, Leigh JA, Heath PJ, Luque I, Tarradas C, Dowson CG, et al. Development of a multilocus sequence typing scheme for the pig pathogen Streptococcus suis: identification of virulent clones and potential capsular serotype exchange. J Clin Microbiol. 2002;40:3671-80.

9. Blume V, Luque I, Vela Al, Borge C, Maldonado A, Dominguez L, et al. Genetic and virulence-phenotype characterization of serotypes 2 and 9 of Streptococcus suis swine isolates. Int Microbiol. 2009;12:161-6.

10. Kerdsin A, Dejsirilert S, Puangpatra P, Sripakdee S, Chumla K, Boonkerd N, et al. Genotypic profiles of Streptococcus suis serotype 2 and clinical features of infections in humans, Thailand. Emerg Infect Dis. 2011;17:835-42.

11. Princivalli MS, Palmieri C, Magi G, Vignaroli C, Manzin A, Camporese A, et al. Genetic diversity of Streptococcus suis clinical isolates from pigs and humans in Italy (2003-2007). Euro Surveill. 2009;14:1-7.

12. Chang B, Wada A, Ikebe T, Ohnishi M, Mita K, Endo M, et al. Characteristics of Streptococcus suis isolated from patients in Japan. Jpn J Infect Dis. 2006;59:397-9.

13. Ye C, Zheng H, Zhang J, Jing H, Wang L, Xiong Y, et al. Clinical, experimental, and genomic differences between intermediately pathogenic, highly pathogenic, and epidemic Streptococcus suis. J Infect Dis. 2009;199:97-107.

14. Ye C, Bai X, Zhang J, Jing H, Zheng H, Du H, et al. Spread of Streptococcus suis sequence type 7, China. Emerg Infect Dis. 2008;14:787-91.

15. Fittipaldi N, Xu J, Lacouture S, Tharavichitkul P, Osaki M, Sekizaki T, et al. Lineage and virulence of Streptococcus suis serotype 2 isolates from North America. Emerg Infect Dis. 2011;17:2239-44.

16. De Greef A, Wisselink HJ, De Bree FM, Schultsz C, Baums CG, Thi HN, et al. Genetic diversity of Streptococcus suis isolates as determined by comparative genome hybridization. BMC Microbiol. 2011;11:161.

17. Fittipaldi N, Gottschalk M, Vanier G, Daigle F, Harel J. Use of selective capture of transcribed sequences to identify genes preferentially expressed by Streptococcus suis upon interaction with porcine brain microvascular endothelial cells. Appl Environ Microbiol. 2007;73:4359-64.

18. Jiang H, Fan HJ, Lu CP. Identification and distribution of putative virulent genes in strains of Streptococcus suis serotype 2. Vet Microbiol. 2009;133:309-16. 
19. Li W, Liu L, Chen H, Zhou R. Identification of Streptococcus suis genes preferentially expressed under iron starvation by selective capture of transcribed sequences. FEMS Microbiol Lett. 2009;292:123-33.

20. Smith HE, Buijs H, De Vries RR, Wisselink HJ, Stockhofe-Zurwieden N, Smits MA. Environmentally regulated genes of Streptococcus suis: identification by the use of iron-restricted conditions in vitro and by experimental infection of piglets. Microbiology. 2001;147:271-80.

21. Vanier G, Fittipaldi N, Slater JD, De La Cruz D-PM, Rycroft AN, Segura M, et al. New putative virulence factors of Streptococcus suis involved in invasion of porcine brain microvascular endothelial cells. Microb Pathog. 2009:46:13-20.

22. Wilson TL, Jeffers J, Rapp-Gabrielson VJ, Martin S, Klein LK, Lowery DE, et al. A novel signature-tagged mutagenesis system for Streptococcus suis serotype 2. Vet Microbiol. 2007;122:135-45

23. Geng H, Zhu L, Yuan Y, Zhang W, Li W, Wang J, et al. Identification and characterization of novel immunogenic proteins of Streptococcus suis serotype 2. J Proteome Res. 2008;7:4132-42.

24. Gómez-Gascón L, Luque I, Olaya-Abril A, Jiménez-Munguía I, Orbegozo-Medina RA, Peralbo E, et al. Exploring the pan-surfome of Streptococcus suis: looking for common protein antigens. J Proteomics. 2012;75:5654-66.

25. Wang $Y$, Dang $Y$, Wang $X$, Lu H, Wang $X$, Lang $X$, et al. Comparative proteomic analysis of Streptococcus suis serotype 2 cell wall-associated proteins. Curr Microbiol. 2011;62:578-88

26. Wang Y, Yi L, Wu Z, Shao J, Liu G, Fan H, et al. Comparative proteomic analysis of Streptococcus suis biofilms and planktonic cells that identified biofilm infection-related immunogenic proteins. PLoS One. 2012;7:e33371.

27. Zhang A, Xie C, Chen $\mathrm{H}$, Jin M. Identification of immunogenic cell wall-associated proteins of Streptococcus suis serotype 2. Proteomics. 2008;8:3506-15.

28. Zhang A, Chen B, Mu X, Zhao Y, Zheng P, Chen $\mathrm{H}$, et al. Identification of three novel in vivo-induced expressed antigens during infection with Streptococcus suis serotype 2. FEMS Microbiol Lett. 2009;295:17-22.

29. Wu Z, Zhang W, Lu C. Comparative proteome analysis of secreted proteins of Streptococcus suis serotype 9 strains from diseased and healthy pigs. Microb Pathog. 2008;45:159-66.

30. Wu Z, Zhang W, Shao J, Wang Y, Lu Y, Lu C. Immunoproteomic assay of secreted proteins of Streptococcus suis serotype 9 with convalescent sera from pigs. Folia Microbiol (Praha). 2011;56:423-30.

31. Jing HB, Yuan J, Wang J, Yuan Y, Zhu L, Liu XK, et al. Proteome analysis of Streptococcus suis serotype 2. Proteomics. 2008;8:333-49.

32. Poon TCW. Opportunities and limitations of SELDI-TOF-MS in biomedical research: practical advices. Expert Rev Proteomics. 2007:4:51-65.

33. Khoder G, Yamaoka Y, Fauchère JL, Burucoa C, Atanassov C. Proteomic Helicobacter pylori biomarkers discriminating between duodenal ulcer and gastric cancer. J Chromatogr B Analyt Technol Biomed Life Sci. 2009;877:1193-9.

34. Lanotte P, Perivier M, Haguenoer E, Mereghetti L, Burucoa C, Claverol S, et al. Proteomic biomarkers associated with Streptococcus agalactiae invasive genogroups. PLoS One. 2013:8:e54393.

35. Bernarde C, Khoder G, Lehours P, Burucoa C, Fauchre JL, Delchier JC, et al. Proteomic Helicobacter pylori biomarkers discriminative of low-grade gastric MALT lymphoma and duodenal ulcer. Proteomics: Clin Appl. 2009;3:672-81.

36. Fagerquist CK, Garbus BR, Miller WG, Williams KE, Yee E, Bates AH, et al. Rapid identification of protein biomarkers of Escherichia coli 0157:H7 by matrix-assisted laser desorption ionization-time-of-flight-time-of-flight mass spectrometry and top-down proteomics. Anal Chem. 2010;82:2717-27125.

37. Maurelli AT. Black holes, antivirulence genes, and gene inactivation in the evolution of bacterial pathogens. FEMS Microbiol Lett. 2007;267:1-8.

38. Zhao G, Zhu L, Feng E, Cao X, Shang N, Liu X, et al. A novel anti-virulence gene revealed by proteomic analysis in Shigella flexneri 2a. Proteome Sci. 2010;8:30

\section{Submit your next manuscript to BioMed Central and take full advantage of:}

- Convenient online submission

- Thorough peer review

- No space constraints or color figure charges

- Immediate publication on acceptance

- Inclusion in PubMed, CAS, Scopus and Google Scholar

- Research which is freely available for redistribution

Submit your manuscript at www.biomedcentral.com/submit 